\section{ORIGINAL RESEARCH}

D.-H. Kang

Y.-H. Hwang

Y.-S. Kim

J. Park

O. Kwon

C. Jung

\title{
Direct Thrombus Retrieval Using the Reperfusion Catheter of the Penumbra System: Forced-Suction Thrombectomy in Acute Ischemic Stroke
}

BACKGROUND AND PURPOSE: Although the PS has been the most promising mechanical thrombectomy device in terms of recanalization rates, even the PS cannot recanalize all cases of occlusion. Under such circumstances, we simply modified the PS, identified certain advantages, and applied this modification as a primary technique for recanalization. Here we describe and discuss the technical details and results of our preliminary experience.

MATERIALS AND METHODS: This study included 22 consecutive patients with acute ischemic stroke secondary to large-artery occlusion who underwent modified thrombectomy by using the PS for recanalization. Direct wedging between the tip of the reperfusion catheter and the proximal part of the clot followed by forceful suction by using a 20 - or $50-\mathrm{mL}$ syringe is a unique feature of this technique. What is distinctive is that this does not require use of a separator or aspiration pump.

RESULTS: All treated vessels (100\%) were successfully recanalized. A $\mathrm{TICl}$ scale of $2 \mathrm{~b}$ or 3 was achieved in $81.9 \%$ of patients. A 3-month favorable functional outcome (mRS score, 0-2) was achieved in $45.5 \%$ of patients. The only procedural complication was a transient dissection of the proximal ICA, which developed while advancing the guide catheter.

CoNCLUSIONS: Forced-suction thrombectomy is a simple modification of the PS. On the basis of our data, this technique allows safe and effective revascularization in acute large-vessel occlusion. Thus, for achieving the best outcome, the modified PS technique is proposed as a viable option for acute stroke management, either by itself or in conjunction with other devices or drugs.

\begin{abstract}
ABBREVIATIONS: $\mathrm{BA}=$ basilar artery; ECASS = European Cooperative Acute Stroke Study; $\mathrm{HI}=$ hemorrhagic infarction; ICA = internal carotid artery; ICH = intracranial hemorrhage; $\mathrm{Lt}=$ left; $\mathrm{MCA}=$ middle cerebral artery; $\mathrm{mRS}=$ modified Rankin scale; NIHSS = National Institutes of Health Stroke Scale; $\mathrm{PH}=$ parenchymal hemorrhage; Post = after; Pre = before; PS = Penumbra System; Rt = right; rtPA = recombinant tissue plasminogen activator; $\mathrm{TICl}=$ Thrombolysis $\mathrm{In}$ Cerebral Infarction
\end{abstract}

$T^{T}$ he impact of early recanalization on outcome in the management of acute ischemic stroke has already been stressed. ${ }^{1,2}$ Focus has recently shifted from pharmaceutical to mechanical methods for recanalization, in an attempt to increase the recanalization rate and reduce the adverse events associated with thrombolytic drugs. ${ }^{3,4}$ Currently available mechanical thrombectomy devices that have received US Food and Drug Administration clearance are the Merci Retriever (Concentric Medical, Mountain View, California) and the PS (Penumbra, Alameda, California), both of which are indicated for use within 8 hours of symptom onset in patients with large intracranial vessel occlusion.

To date, the most promising device in terms of recanalization rate is the PS, which is designed for continuous disruption and aspiration of thrombi by using a separator, reperfusion catheter,

Received May 24, 2010; accepted after revision July 22.

From the Departments of Neuroradiology (D.-H.K., Y.-S.K.), Neurosurgery (J.P.), and Neurology (Y.-H.H.), Daegu-Gyeongbuk Cardiocerebrovascular Center, Kyungpook National University, Daegu, Republic of Korea; and Departments of Neurosurgery (O.K.) and Radiology (C.J.), Stroke Center, Seoul National University Bundang Hospital, Seongnam, Republic of Korea

D.-H.K. and Y.-H.H. contributed equally to this study.

Please address correspondence to Yong-Sun Kim, MD, Department of Neuroradiology, Daegu-Gyeongbuk Cardiocerebovascular Center, Kyungpook National University Hospital, 50, Samduk-2-ga, Jung-gu, Daegu, Republic of Korea 700-21; e-mail: knuhnr@hotmail.com

Indicates open access to non-subscribers at www.ajnr.org

DOI 10.3174/ajnr.A2299 and aspiration pump. ${ }^{3}$ However, revascularization is not always possible despite use of the PS, particularly when the clot is so hard that the separator will not advance or if the clot is located in a tortuous segment of the vessel, in which case advancement of the separator can cause injury to the surrounding endothelium. In our early experience with the PS, we failed to recanalize a few cases due to the aforementioned situations; thus, we modified the standard PS technique. The modification resulted in successful recanalization. Thereafter, the modified PS technique, called "forcedsuction thrombectomy," has been applied to patients with major stroke admitted to our institutions. The purpose of this study was to describe the technical details and to evaluate the safety and efficacy of the modified PS technique.

\section{Materials and Methods}

Since March 2009, the modified PS technique has been applied to 22 consecutive patients with acute ischemic stroke following large intracranial arterial occlusions within 8 hours of symptom onset. We applied the present technique as a primary one for mechanical revascularization. In case of failure, we planned a conversion to the standard PS technique or other interventions.

Inclusion and exclusion criteria for this study were similar to those of previous mechanical thrombectomy trials. ${ }^{3,4}$ We included patients who had clinical signs and symptoms consistent with acute ischemic stroke, simultaneously showed occlusion of a treatable intracranial vessel, and could be treated within 8 hours from symptom onset. 
Within the 3-hour time window, we performed this technique in patients with persistent major arterial occlusions on conventional angiography despite intravenous rtPA. Selection of the candidates for conventional angiography was based on major arterial occlusions on MR angiography following intravenous rtPA. We excluded patients who were at risk of bleeding (ie, infarction volume more than half of the affected vascular territory) or revealed severe brain edema, ICH, or uncontrolled hypertension.

The safety of the present technique was evaluated according to the incidence of procedural complications based on findings from procedure angiography, immediate postprocedural CT scans, and neurologic examinations. Neurologic status was evaluated by a neurologist by using the NIHSS score before and immediately following the procedure. Procedural complications were defined as vascular perforation, intramural arterial dissection, and embolization of previously uninvolved territory. Histologic examination of the retrieved clots was performed to determine whether the clots contained endothelial components in cases of difficult procedures. Additional CT scans were obtained to detect ICH at 24-48 hours and when the subject showed symptoms indicative of ICH based on the attending physician's decision-making. If there was evidence of ICH, the ECASS II classifications for ICH were used for this study: HI 1, 2, and PH 1, $2 .^{5}$ Symptomatic ICH was further defined by CT evidence of ICH associated with more than a 4-point deterioration of the NIHSS score. In addition, patients were evaluated for neurologic and functional status by using the NIHSS and mRS scores at 7 days, 30 days ( \pm 10 days), and 90 days ( \pm 10 days) after treatment.

Efficacy of this technique was assessed by the incidence of successful revascularization of the target vessel following the technique. Successful recanalization was defined as TICI grade 2 or 3 . The interval from arterial puncture to recanalization was recorded in minutes.

\section{Description of the Modified PS Technique}

After obtaining signed informed consent, we performed an arterial puncture with the patient under general anesthesia and systemic heparinization. Four-vessel digital subtraction angiography was performed to define the surrounding angioarchitecture of the occluded vessel. Then, the double coaxial system, which was assembled by an outermost 80-cm-long 6F introducer sheath (Shuttle-SL guide sheath; Cook, Bloomington, Indiana) and an inner 90- or 100-cmlong 6F guide catheter (Envoy; Cordis, Miami Lakes, Florida), was placed in the territory of the target vessel to enable approach of the PS reperfusion catheter. We advanced the PS reperfusion catheter to the proximal end of the thrombus to wedge the catheter with the clot. A 1.7F microcatheter was introduced coaxially beyond the occlusion, and local angiography was performed before reperfusion therapy to predict the original path of the occluded vessel and to inspect the outline of the occlusion, including the size of the clot. This process could be skipped if the clot was too hard to pass the microguidewire or microcatheter.

After withdrawal of the $1.7 \mathrm{~F}$ microcatheter, we connected a $20-\mathrm{mL}$ syringe to the proximal hub of the reperfusion catheter (Fig 1E). At this time, we paid attention to 3 points: First, we used the largest possible reperfusion catheter to maximize suction power; second, we checked the direction of the tip of the reperfusion catheter to keep it parallel to the presumptive path of the occluded vessel for prevention of direct contact between the tip and the endothelium; and third, we confirmed direct wedging and a vacuum state between the tip and the clot by slightly pulling on the syringe. Thereafter, we pulled the plunger forcefully to enable the reperfusion catheter to function as a vacuum pad to the clot and gradually withdrew the catheter, maintaining the pulling forces. The procedure resulted in 1 of 2 types of conditions. First, the vacuum state was unexpectedly lifted, followed by entry of blood flow into the catheter, suggesting that the wedged clot was disrupted and sucked into the catheter (disrupted-clot type, Fig $1 F$ ). We then repeated the same procedure while advancing the reperfusion catheter by degrees. Second, the vacuum state was maintained until the reperfusion catheter was finally withdrawn from the guide catheter, implying that whole clot was wedged and retrieved from the occluded vessel (whole-clot type, Fig $1 G$ ). Immediate reperfusion was achieved in most of these conditions. If we failed to recanalize the occluded vessel by using a $20-\mathrm{mL}$ syringe, we changed to a $50-\mathrm{mL}$ syringe to intensify the suction power.

\section{Results}

Clinical and radiologic characteristics of a total of 22 subjects are shown in the Table. The study included 14 men and 8 women; the mean age was $59 \pm 12$ years. The mean interval from the onset of symptoms to arterial puncture was $5.3 \pm 1.9$ hours, and baseline NIHSS was $18.1 \pm 5.2$. Fourteen of the 22 subjects $(63.6 \%)$ were treated with intravenous rtPA within a 3-hour time window. Target-vessel locations were 14 MCAs (63.6\%), 4 ICAs (18.2\%), and 4 BAs (18.2\%). Etiologic stroke classification showed that cardioembolism was the most common in this study (63.6\%). All treated target vessels were TICI 0 before the procedure; subsequently, all cases (100\%) were successfully recanalized to TICI $2 \mathrm{a}(4 / 22,18.1 \%), 2 \mathrm{~b}(10 / 22$, $45.5 \%)$ or $3(8 / 22,36.4 \%)$ following the presented technique. The mean procedure duration from arterial puncture to revascularization including diagnostic angiography was $40.2 \mathrm{~min}$ utes, and, notably, within 30 minutes in 10 cases $(45.5 \%)$. Moreover, 9 of these rapidly revascularized cases were the whole-clot type. In every patient, we first used a 041 Penumbra reperfusion catheter for the procedure and additionally used a 032 Penumbra reperfusion catheter in 2 cases of distal embolization to the M2 segment of the MCA.

Adjuvant procedures were performed in 4 patients. In 1 patient, we performed balloon angioplasty due to the underlying stenosis of the BA following revascularization of the BA occlusion. In the other 3 patients, emergency stent placement of the proximal segment of the ICA was performed to provide access to a reperfusion catheter. In 1 procedural complication, proximal ICA dissection occurred while we were placing the guide catheter in the tortuous vessel, and recovery was confirmed on follow-up MR angiography 5 days after the procedure. Histologic examination of the retrieved clots from 2 cases of difficult procedures resulted in no endothelial component in the clots. ICH occurred in 7 patients (31.8\%): 2 HI-1, 3 HI-2, 1 PH-1, and 1 PH-2. Two (9.1\%) patients were symptomatic, and 1 underwent decompressive surgery due to accompanying edema, while the other showed severe deterioration immediately and later died. The favorable functional outcome (mRS, $0-2$ ) at 3 months was $45.5 \%$ ( 10 of 22 cases): mRS 0 , four; mRS 1, two; and mRS 2, four. The all-cause mortality was $13.6 \%$ in this study: 2 cases of malignant brain edema and 1 recurrent ischemic stroke during follow-up.

\section{Illustrative Case}

A 45-year-old man (patient 22) had sudden-onset global aphasia and right hemiparesis. CT findings were normal, and 

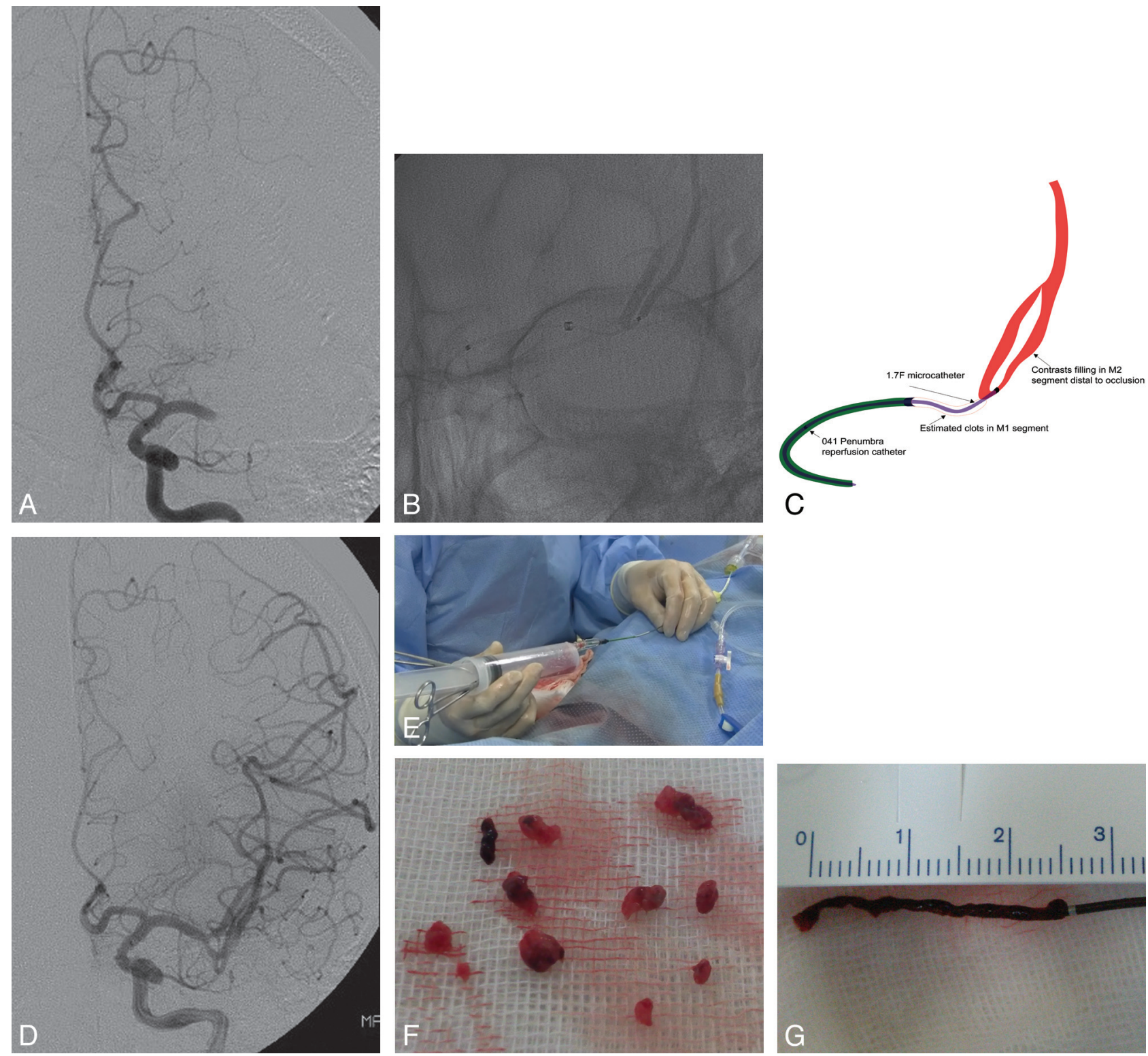

Fig 1. Case 22. A, Pretreatment angiography shows total occlusion of the M1 segment of the left MCA. $B$ and $C$, Angiogram and illustration of the procedure show the occlusion site and surrounding angioarchitecture. D, Immediate postprocedural angiography shows complete (TICl 3) revascularization. E, Photograph shows that the 20- or 50-mL syringe is connected to the proximal hub of the reperfusion catheter with forceful suction. F, Disrupted clot. $G$. Whole clot retrieved from the occlusion of patient 22 is shown.

carotid angiography demonstrated complete occlusion (TICI 0 ) of the proximal M1 segment of the left MCA (Fig 1A). On the basis of a diagnosis of cardioembolic stroke, we performed immediate recanalization by using the modified PS technique 3 hours after onset of symptoms. We placed the guide catheter on the cervical segment of the ICA, and followed by advancing the 041 Penumbra reperfusion catheter to the proximal part of the clot. Then, a microcatheter was introduced beyond the thrombus, and local angiography was performed for inspection of the occluded segment (Fig $1 B,-C$ ). After wedging the reperfusion catheter to the proximal part of the clot, a single forceful suction with a $50-\mathrm{mL}$ syringe enabled retrieval of the whole clot (Fig $1 G$ ) and resulted in a TICI 3 recanalization (Fig $1 D)$. The interval from arterial puncture to revascularization was 15 minutes. No procedure-related complications or ICH occurred, and the patient improved in NIHSS score, from 20 at baseline to 14 at 1 day after the procedure.

\section{Discussion}

\section{Advantages and Precautions of the Modified PS Technique}

Although the Penumbra Phase 1 Trial showed a 100\% revascularization rate to Thrombolysis in Myocardial Infarction grade of 2 or 3, the pivotal Penumbra Phase 2 Trial recently reported an overall revascularization rate of $81.6 \%{ }^{3}$ Also, in our experience with the standard PS, recanalization was not achieved in a few cases, particularly in the case of very firm thrombi and/or occlusion of a severely tortuous segment. This experience prompted us to modify the technique while adopting as many of the advantages of the PS as possible, which included a wider but easily navigable reperfusion catheter and the concept of suction for revascularization. The modifications were simple and intuitive: First, use of a reperfusion catheter tip as a vacuum pad for the thrombus; and second, 


\begin{tabular}{|c|c|c|c|c|c|c|c|c|c|}
\hline \multirow[b]{2}{*}{ No. } & \multirow{2}{*}{$\begin{array}{l}\text { Time to } \\
\text { Arterial } \\
\text { Puncture } \\
\text { (h) }\end{array}$} & \multirow{2}{*}{$\begin{array}{c}\text { Target } \\
\text { Vessel } \\
\text { Location }\end{array}$} & \multirow[b]{2}{*}{$\begin{array}{c}\text { Baseline } \\
\text { NIHSS }\end{array}$} & \multicolumn{2}{|c|}{$\mathrm{TICl}$} & \multirow{2}{*}{$\begin{array}{c}\text { Puncture to } \\
\text { Recanalization } \\
\text { Time (min) }\end{array}$} & \multirow[b]{2}{*}{$\begin{array}{l}\text { Intravenous rtPA } \\
\qquad(\mathrm{mg} / \mathrm{kg})^{\mathrm{a}}\end{array}$} & \multirow[b]{2}{*}{$\begin{array}{l}\text { Hemorrhagic Event According } \\
\text { to ECASS Classifications }{ }^{5}\end{array}$} & \multirow[b]{2}{*}{$\begin{array}{l}\mathrm{mRS} \text { at } \\
90 \text { Days }\end{array}$} \\
\hline & & & & Pre & Post & & & & \\
\hline- & 8.5 & BA & 23 & 0 & $2 b$ & 80 & - & None & 1 \\
\hline 2 & 3 & Lt M1 & 24 & 0 & $2 b$ & 30 & 0.6 & None & 2 \\
\hline 3 & 3 & Rt M1 & 19 & 0 & $2 b$ & 15 & 0.6 & None & 1 \\
\hline 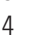 & 4 & $\mathrm{BA}$ & 24 & 0 & $2 a$ & 80 & 0.6 & Asymptomatic HI-2 & 5 \\
\hline 5 & 4.5 & Rt M1 & 16 & 0 & $2 b$ & 40 & 0.6 & None & 3 \\
\hline 6 & 5.5 & Rt ICA & 16 & 0 & $2 a$ & 50 & 0.9 & Asymptomatic HI-1 & 2 \\
\hline 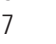 & 6 & Rt. M1 & 14 & 0 & $2 b$ & 30 & - & None & 0 \\
\hline 8 & 2.5 & Rt. M1 & 15 & 0 & 3 & 40 & 0.6 & None & 5 \\
\hline 9 & 2.5 & Lt ICA & 21 & 0 & 3 & 25 & 0.6 & None & 6 \\
\hline 10 & 6 & Rt ICA & 16 & 0 & $2 \mathrm{a}$ & 60 & 0.9 & None & 3 \\
\hline 11 & 5.5 & Rt ICA & 16 & 0 & 3 & 40 & - & None & 3 \\
\hline 12 & 4 & BA & 29 & 0 & $2 a$ & 60 & - & Asymptomatic HI-2 & 5 \\
\hline 13 & 5.5 & Lt M1 & 23 & 0 & $2 b$ & 50 & 0.9 & None & 6 \\
\hline 14 & 4 & Lt M1 & 11 & 0 & 3 & 60 & 0.6 & None & 4 \\
\hline 15 & 8 & BA & 25 & 0 & $2 b$ & 50 & - & Symptomatic PH-2 & 6 \\
\hline 16 & 7 & Lt M1 & 20 & 0 & 3 & 15 & 0.6 & Symptomatic PH-1 & 3 \\
\hline 17 & 6 & Rt M1 & 10 & 0 & 3 & 20 & - & None & 0 \\
\hline 18 & 6 & Lt M1 & 12 & 0 & $2 b$ & 10 & 0.6 & None & 0 \\
\hline 19 & 5.5 & Rt M1 & 11 & 0 & $2 b$ & 30 & - & None & 0 \\
\hline 20 & 7 & Lt M1 & 18 & 0 & $2 b$ & 50 & - & Asymptomatic HI-2 & 3 \\
\hline 21 & 9 & Lt M1 & 14 & 0 & 3 & 30 & 0.6 & Asymptomatic HI-1 & 2 \\
\hline 22 & 3 & Lt M1 & 20 & 0 & 3 & 15 & 0.9 & None & 2 \\
\hline
\end{tabular}

- indicates dosage of rtPA.

intensification of suction power via forceful pulling of syringe plunger. The modified PS technique provided several benefits: First, it is a simplification of the standard PS; thus, no additional manipulation or equipment is required and preparation time of the procedure is also shorter. Second, revascularization can be achieved faster because the technique is brief; and third, if failure occurs, conversion to the standard PS technique or other procedures is easy.

This technique can be applied variably in clinical practice. We placed the present technique before the standard PS method. However, others can use this technique as a rescue or adjuvant procedure in case of failure to recanalize by the standard PS or other interventions. Direct wedging between the catheter tip and thrombus is a feature of this technique that requires some attention. Reinforced suction power can injure the surrounding endothelium when the tip directly contacts the vessel wall; therefore, careful interpretation of proximal and distal angiography is needed to predict the original path of the occluded artery along with trying to keep the tip of the reperfusion catheter parallel to the estimated path. In our practice, a poor catheter position was identified in 2 cases of ICA occlusion, which was located just beyond the curve of the carotid siphon. In these cases, the catheter tip was directed to the dorsal wall of distal ICA because of the tortuous anatomy of the carotid siphon. However, this could be overcome without a problem by primary steam-shaping of the tip of the reperfusion catheter. In addition, slight pulling the plunger just before forceful suction is also required to confirm and strengthen direct wedging.

\section{Efficacy and Safety of the Modified PS Technique}

In this study, the modified PS technique resulted in $100 \%$ recanalization (TICI $\geq 2$ ) and $45.5 \%$ favorable functional out- come, which is comparable with or favorable to the previous standard PS technique. ${ }^{3,6}$ The mean procedural time for this modified technique of 40.2 minutes is notable and was within 30 minutes in 10 cases (45.5\%). Moreover, 9 of these rapidly recanalized cases were the whole-clot type, which suggests that clot disruption is not always required for aspiration in some cases of large intracranial vessel occlusion. Short procedural time and rapid revascularization in the present study could potentially increase the advantages of this technique with regard to the concept of timely revascularization in acute stroke therapy. $^{7}$

Only 1 procedural complication (4.5\%), transient dissection on the cervical ICA, occurred while advancing the guide catheter into the tortuous segment. This complication rate seemed to be comparable or favorable when compared with published rates of other interventions, including previous Penumbra trials. ${ }^{2-4}$

On the basis of 24-hour CT scans, 7 subjects were found to have ICH in this study; 2 of these $(9.1 \%)$ were symptomatic and $5(22.7 \%)$ were asymptomatic. This hemorrhage rate also seemed comparable with published rates for stroke cohorts with similar demographics and time windows. ${ }^{3,4,7}$

\section{Limitations}

First, the major limitation of this study is that we did not compare the modified technique with the standard PS. However, we could not compare the techniques because of our limited experience with the standard PS. Moreover, the focus of this study was a viable technical option in rescue situations rather than the comparison of 2 techniques. Second, despite angiographic confirmation of major intracranial arterial occlusion before the modified PS technique, the results of $100 \%$ recanalization should be interpreted cautiously because more 
than half of patients received intravenous rtPA before the procedure, which might affect the clot lysis during the procedure. Finally, because this study was based on the limited experience of 2 centers and only covers a relatively small series, it lacks sufficient power to confirm the safety and efficacy of this modified thrombectomy technique. Thus, to prove the clinical benefits of the proposed endovascular thrombectomy technique, a larger prospective multi-institutional study is necessary.

\section{Conclusions}

The present technique is a simple modification of the PS, which simultaneously makes the best use of this system. Our preliminary experience demonstrates that the modified PS technique allows safe and effective revascularization in patients with acute ischemic stroke secondary to large-vessel occlusion. It is simple and intuitive and can allow faster revascularization of the occluded vessel; with failure, it can be easily converted to the standard PS method or other interventions. Thus, the present technique is proposed as a viable option for acute stroke management, either by itself or in conjunction with other devices or drugs for achievement of the best outcome.

\section{References}

1. Jansen O, Schellinger P, Fiebach J, et al. Early recanalisation in acute ischaemic stroke saves tissue at risk defined by MRI. Lancet 1999;353:2036-37

2. Rha JH, Saver JL. The impact of recanalization on ischemic stroke outcome: a meta-analysis. Stroke 2007;38:967-73

3. Penumbra Pivotal Stroke Trial Investigators. The Penumbra Pivotal Stroke trial: safety and effectiveness of a new generation of mechanical devices for clot removal in intracranial large vessel occlusive disease. Stroke 2009;40: 2761-68. Epub 2009 Jul 9

4. Smith WS, Sung G, Saver J, et al. Mechanical thrombectomy for acute ischemic stroke: final results of the Multi MERCI trial. Stroke 2008;39:1205-12. Epub $2008 \mathrm{Feb} 28$

5. Hacke W, Kaste M, Fieschi C, et al. Randomised double-blind placebo-controlled trial of thrombolytic therapy with intravenous alteplase in acute ischaemic stroke (ECASS II): Second European-Australasian Acute Stroke Study Investigators. Lancet 1998;352:1245-51

6. Grunwald IQ, Walter S, Papanagiotou P, et al. Revascularization in acute ischaemic stroke using the Penumbra system: the first single center experience. Eur J Neurol 2009;16:1210-16

7. Furlan A, Higashida R, Wechsler L, et al. Intra-arterial prourokinase for acute ischemic stroke: the PROACT II study—a randomized controlled trial. Prolyse in Acute Cerebral Thromboembolism. JAMA 1999;282:2003-11 